\title{
Immunosuppressive therapy for chronic uveitis: optimising therapy with steroids and cyclosporin A
}

\author{
Andrew D Dick, Mohammed Azim, John V Forrester
}

Uveitis denotes inflammation of the uveal tract of the eye and is generally categorised as either anterior (involving the iris/ciliary body) or posterior (involving the choroid as well as adjacent structures including pigment epithelium and retina). ${ }^{1}$ Although uveitis is a known cause of blindness, until recently there were few data reflecting the prevalence and incidence of uveitis among the blind, mainly because of the lack of information concerning the incidence of sight threatening complications from uveitis. One recent study has shown that the incidence of visual impairment in uveitis is $35 \%$ and is mainly due to posterior uveitis, largely as a result of persistent cystoid macular oedema. ${ }^{2}$ Other studies have shown that there is a $25 \%$ loss of vision in $4 \%$ of patients with anterior uveitis, $43 \%$ with posterior uveitis, and $40 \%$ with panuveitis. ${ }^{3}$ Moreover, specific uveitic syndromes-for example, Behçet's disease $^{45}$ and serpiginous chorioretinopathy, ${ }^{6}$ have a renowned poor prognosis without therapy. SuttorpSchulten and Rothova ${ }^{7}$ have highlighted the socioeconomic impact of blindness due to uveitis, especially as most cases of visual impairment occur in the working age group, and uveitis now ranks with diabetic retinopathy as one of the major causes of visual handicap in this age group. As many uveitic entities are curable, or at least controllable in the sense that ocular damage can be prevented or limited with early and adequate therapy, we discuss a structured and rational approach to immunosuppression with respect to the control of intraocular inflammation, which aims to maintain best possible visual function while minimising therapy induced side effects, in particular those which occur with prolonged steroid therapy.

\section{Immunopathogenesis: directing therapy}

INFECTIOUS VERSUS NON-INFECTIOUS UVEITIS

Fifty per cent of cases of uveitis are classified as idiopathic ${ }^{12}$ while many of the others are associated with or form part of other disease entities. However, before considering and commencing immunosuppressive therapy for uveitis, direct infectious causes must be excluded so that appropriate therapy can be commenced. Therefore, management of endogenous posterior uveitis depends on both clinical and associated laboratory based investigations to exclude any infectious aetiology. Many cases of infectious uveitis are clinically obvious-for example, opportunistic infections in immunocompromised individuals (especially in association with HIV) are increasingly frequent causes of uveitis, the treatment of which is highly dependent on specific antimicrobial therapy. In non-immunocompromised individuals, toxoplasmosis (depending on which country and region) is one of the leading causes of uveitis, ${ }^{28}$ and although immunosuppression is used to treat this condition it must be used in conjunction with appropriate antibiotic therapy to reduce the incidence of disease relapse. In certain cases, however, differentiation between infectious and non-infectious endogenous posterior uveitis (EPU) may be more difficult. For example, Lyme disease, miliary tuberculosis, or progressive outer retinal necrosis (PORN), may present with signs identical to those of EPU and, despite adequate clinical assessment, immunosuppressive therapy may have already been started on the basis that the condition was considered noninfectious. In these cases, as well as possible 'masquerade' conditions such as ocular lymphoma a failure to respond should be a warning sign that the diagnosis may be incorrect and needs to be reviewed. Patients with true EPU are assumed to be autoimmune in nature, ${ }^{19}$ and the aim of this review is to focus on the treatment of these primary immune mediated conditions.

IMMUNE MECHANISMS IN UVEITIS

Endogenous anterior and posterior uveitis are clinically and pathogenetically distinct diseases. Most cases of anterior uveitis are acute, self limiting diseases that respond rapidly to therapy and are probably mediated by major histocompatibility complex (MHC) class $\mathrm{I} / \mathrm{CD}^{+}$cytotoxic mechanisms. ${ }^{1}$ In contrast, endogenous posterior uveoretinitis (EPU) comprises chronic, relapsing disorders that progressively damage vision and are $\mathrm{MHC}$ class II/CD $4^{+} \mathrm{T}$ cell mediated diseases. ${ }^{1011}$ There is clinically a degree of overlap in presentation and course of disease-for example, juvenile rheumatoid arthritis and EPU, despite the pathogenic differences. However, in either condition if significant sight threatening disease is present a common approach of immunosuppression, as we will discuss, should be commenced. While there is no definitive evidence that putative target (auto)antigens, which include $\mathrm{S}$-antigen (S-Ag) and interphotoreceptor retinoid binding protein (IRBP), ${ }^{12}$ are directly involved in the induction of EPU, the experimental models have shown that EPU is initiated by antigen specific $\mathrm{CD} 4^{+} \mathrm{T}$ cells ${ }^{13}{ }^{14}$ and can be inhibited by the immunosuppressant cyclosporin $\mathrm{A}$ (CsA).$^{15}$ Furthermore, in EPU activation of peripheral $\mathrm{T}$ cells and other markers of immune activation can be detected. ${ }^{16}{ }^{17}$ Compellingly, there is preliminary evidence that oral tolerance with S-Ag may suppress human EPU. ${ }^{18}$ Currently, however, the most specific and successful method of treating non-infectious EPU is immunosuppression based on inhibiting the activation of $\mathrm{CD} 4^{+} \mathrm{T}$ cells with CsA as documented several years ago. ${ }^{19}$

Non-infectious EPU may occur in isolation or as a feature of systemic autoimmune disease-for example, systemic vasculitis, systemic lupus erythematosus, polyarteritis nodosa, seronegative spondyloarthritides, Behçet's disease, sarcoidosis, multiple sclerosis, to name a few. Immunosuppression in these circumstances must be tailored to treat both the ocular disease as well as systemic 
disease. Successful immunosuppression for ocular inflammation in these conditions will be achieved using the same approach as for idiopathic EPU, especially if the systemic component is quiescent. However, the treatment of sight threatening ocular disease does not depend on whether there is systemic signs of disease or not and should not be governed by the presence or absence of clinical signs. If a significant systemic component is present then immunosuppression must be tailored to treat both ocular and systemic signs.

\section{Indications for treatment of endogenous posterior uveitis}

WHAT TO TREAT?

The appropriate treatment and choice of immunosuppressive therapy for uveitis depends on both the extent and severity of the inflammatory process. Firstly, it is important to establish whether immunosuppression is necessary and, secondly, whether the patient's general medical condition can tolerate immunosuppression. Although numerous reports on the management of idiopathic uveitis ${ }^{20} 21$ describe the use of immunosuppressive therapy, ranging from orbital floor steroids and systemic steroids to newer agents such as CsA and other cytotoxic agents, the long term use of immunosuppressants by ophthalmologists is still not widespread for several reasons:

- many cases in which steroids have been tried are considered 'treatment failures' or non-responders, when relapse occurs on lowering the dose or when significant side effects occur after prolonged use. Such cases are not, in fact, treatment 'failures' since steroid therapy was effective albeit at an unacceptably high dose. However, they do highlight the fact that steroids are not appropriate immunosuppressives in the long term.

- many patients are treated too late when visual recovery is unlikely owing to apparent retinal and/or optic nerve damage. Immunosuppressive therapy thus acquires a reputation for being ineffective. Despite this, it is remarkable how often an irreversibly 'damaged' macula may recover some function.

- apart from distinct uveitic syndromes where immunosuppression has been shown to be beneficial-for example, retinal vasculitis and Behçet's disease, ${ }^{4-6}$ the majority of cases are not easily grouped into such classic diagnoses and the nature of their sight threatening disease is not recognised.

EPU represents a spectrum of autoimmune ocular inflammatory disease normally manifesting a set of clinical features - namely vitritis, chorioretinitis, retinal vasculitis, macula, and disc oedema and less commonly retinochoroidal neovascularisation. ${ }^{9}{ }^{12} 22$ The severity and distribution of these clinical features will determine both whether to and which immunosuppressive(s) to commence. Since EPU is immune mediated and generated systemically attempts to treat uniocular disease with, for instance, periocular steroids alone will lead to less than optimal control of disease thus prolonging any damaging effects in the affected eye and possibly permitting an earlier onset of disease in the fellow eye by failing to suppress systemically activated cytotoxic $\mathrm{T}$ cells. ${ }^{16}$

The decision to treat is determined therefore by the severity of inflammation, classified according to the extent and degree of inflammatory eye signs ${ }^{22}$ as minimal, mild, moderate, and severe. The features which characterise these grades are described below, and are based on detecting 'sight threatening' inflammation:

- minimal disease occurs when there are one or two of the cardinal features of inflammation (see above), such as occasional chorioretinal infiltrates (white dots), minimal or no peripheral vasculitis, and minimal vitritis. The macula/optic nerve is not involved at this stage.

- mild disease occurs when there are more signs of inflammation and these are more extensive-for example, focal active vasculitis, more numerous chorioretinal infiltrates, and vitritis. The macula and optic nerve again are not involved at this stage.

- moderate disease is present when most of the inflammatory features such as dense vitritis are present and, in addition, there is involvement of or imminent threat to the macula or optic nerve. Significant retinal vessel inflammation is usually present. Sight is threatened or already impaired because of, for example, macular oedema. It is important to recognise that sight threatening disease, involving the optic nerve and macula, may be present without many other ocular inflammatory signs.

- severe disease is characterised by extensive changes in any of the cardinal features all of which are present- in particular, chorioretinal inflammation, retinal vasculitis (especially arteritis), optic neuropathy, and vitritis. Vision is usually impaired but may still be normal although under imminent threat because of active macular and/or optic nerve disease.

WHEN TO TREAT

Treatment should be commenced when sight threatening inflammation is present-for example, optic nerve swelling and central retinal vasculitis in Behçet's disease, active serpiginous lesions threatening the fovea, a large central choroidal lesion in multifocal choroiditis, cystoid macular oedema in intermediate uveitis, or a subretinal neovascular membrane in 'ocular histoplasmosis'. With respect to the clinical signs and stages of intraocular inflammation described above, the threat to sight may be considered to be remote, possible, probable, or likely, and this will determine not only when it is appropriate to commence immunosuppressive therapy but also how quickly and which agents to use.

\section{Therapeutic options for treatment of endogenous posterior uveitis}

Experimentally there are many routes whereby EPU can be suppressed, inhibiting either the afferent or the efferent immune response. ${ }^{23}$ These novel approaches presage a future for more specific therapy for EPU..$^{23-25}$ Some of these approaches are already used for refractory cases of autoimmune diseases-for example, monoclonal antibody therapy directed against $\mathrm{CD}^{+} \mathrm{T}$ cells for rheumatoid arthritis and uveitis (CDw52 (Campath)) and tumour necrosis factor (TNF) cytokine receptor fusion protein of deleting anti-TNF monoclonal antibodies for rheumatoid arthritis. ${ }^{26-28}$ Although in their infancy these developments are increasing the range of possible treatments which have the potential to secure longer term immunoregulation, and may be directed toward ophthalmic use (see below).

Clinically, the mainstay of immunosuppressive therapy for EPU over the past two to three decades has been systemic steroids, ${ }^{29}$ but treatment schedules have been erratic and prolonged therapy has rarely been possible because of severe side effects. Despite significant toxicity induced by immunosuppressants during the past 12 years CsA has been used for the treatment of EPU with significant benefit. CsA arguably represents the drug of choice for the long term treatment of sight threatening EPU, because with judicious use toxicity can be minimised. ${ }^{19}{ }^{30-33}$ In addition, costs may restrict early use of CsA in favour of other immunosuppressives. In the following sections a brief discussion of the main immunosuppressive drugs used for EPU is presented. 
Table 1 Summary of diagnosis and treatment regimens in a cohort of patients with endogenous posterior uveitis at the ocular inflammatory clinics in Aberdeen. A diagnosis of idiopathic consists of patients with undefined posterior uveitis and panuveitis, which includes diagnosis such as presumed ocular histoplasmosis syndrome and others where posterior segment features continually change or cannot be specified to other diagnostic descriptions (for example, overlap syndromes)

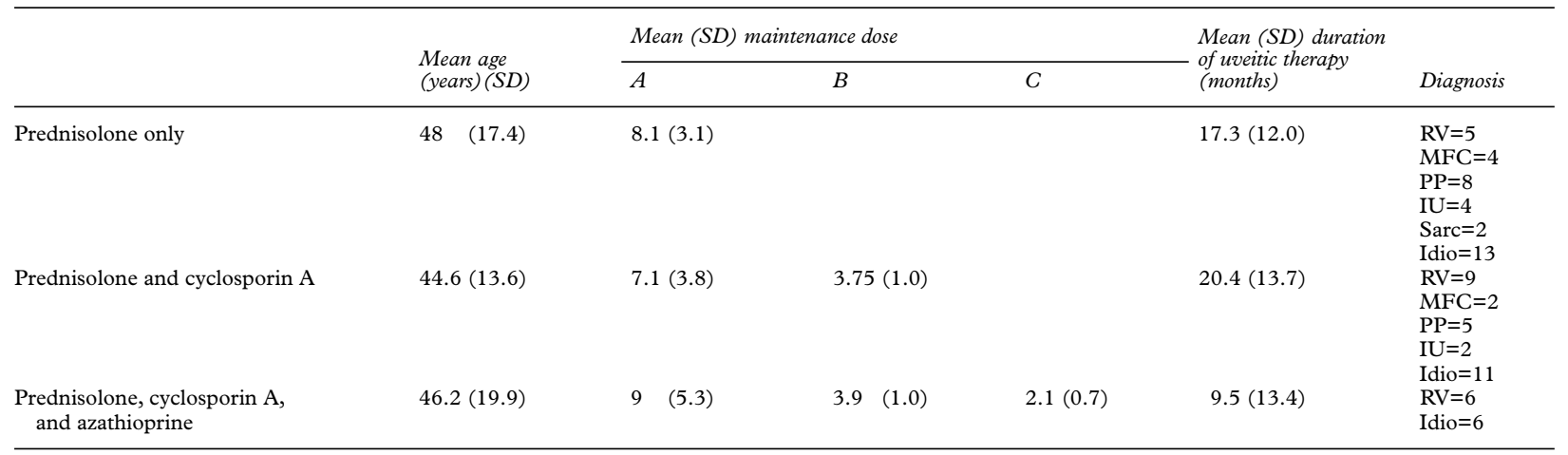

Maintenance doses: $(\mathrm{A})=$ prednisolone $(\mathrm{mg} / \mathrm{day}),(\mathrm{B})=$ cyclosporin $\mathrm{A}(\mathrm{mg} / \mathrm{kg} / \mathrm{day}),(\mathrm{C})=$ azathioprine $(\mathrm{mg} / \mathrm{kg} / \mathrm{day})$

Uveitic diagnoses include: $\mathrm{RV}=$ retinal vasculitis; $\mathrm{MFC}=$ multifocal choroiditis; $\mathrm{PP}=$ pars planitis; $\mathrm{IU}=$ intermediate uveitis; Sarc $=$ sarcoidosis; Idio = idiopathic.

\section{CORTICOSTEROIDS}

Steroids may be administered by several routes and traditionally uniocular, mild intraocular inflammation is treated with orbital floor steroids since it is thought that systemically induced side effects can be avoided. ${ }^{34}{ }^{35}$ However, steroids administered by this route are absorbed systemically, producing serum levels comparable with those achieved with $50 \mathrm{mg}$ of oral prednisolone ${ }^{36}$ and, furthermore, the effect soon wears off because a single dose of 50 mg parenteral prednisolone is not adequate to control inflammation long term. Toxicity with orbital floor steroids is undoubtedly less but mainly for dose related reasons and not because of reduced absorption. Although others use orbital floor steroids we believe that systemic (oral) steroids are usually required for safe control of mild/ moderate ocular inflammation whether the disease is unilateral or bilateral. ${ }^{20}{ }^{21}$ High dose steroids are also effective for the short term treatment of severe inflammation. ${ }^{37}$ However, as Howe et al showed ${ }^{37}$ patients with EPU require therapy for at least 12 months to maintain control with a high incidence $(50 \%)$ of side effects $(18 \%$ therapeutically significant) while $40 \%$ required additional immunosuppression. Therefore, long term treatment of EPU with steroids alone has to be viewed with considerable caution as the therapeutic window which avoids side effects without the risk of relapse is small even though corticosteroids and cyclosporin are equally effective when used as monotherapy for mild to moderate disease. ${ }^{38}$

Table 1 presents a retrospective review of a cohort of patients treated at Aberdeen Royal Hospitals NHS Trust from 1992 to 1996, with either prednisolone alone, or combined with CsA or CsA and azathioprine. Within each group, patients presented with varying diagnoses of EPU. Figure 1 shows the visual recovery in 36 patients who on presentation had mild or moderate ocular inflammation and a mean visual acuity at presentation of $6 / 12$ (in $35 / 53$ eyes) and were treated with a reducing course of prednisolone and then a maintenance dose of $10 \mathrm{mg} /$ day or less. In the majority of patients either visual recovery was observed or acuity was maintained (37/53 affected eyes) and in 33 patients steroids were eventually stopped while maintaining post-treatment vision.

\section{CYCLOSPORIN A}

CsA was first introduced for the prevention of renal allograft rejection. ${ }^{39} \mathrm{CsA}$ is a powerful immunosuppressant which works by inhibiting calcium dependent T cell receptor (TCR) mediated signal transduction and thus activation of $\mathrm{T}$ cells. ${ }^{40}$ This also leads to a down regulation of IL-2 gene transcription ${ }^{41}$ and IL-2 receptor expression.
Since the initial reports indicating that CsA suppressed $\mathrm{EAU}^{15}$ and the later demonstration of its clinical value in treating steroid and cytotoxic resistant intraocular inflammation, ${ }^{19}$ it has now been used for the treatment of most forms of EPU. ${ }^{30-33} 4243$

Figure 2 shows the response of treatment of patients with moderate or severe intraocular inflammation (see above) treated with combined low dose CsA ( $5 \mathrm{mg} / \mathrm{kg} /$ day) and prednisolone ( $<10 \mathrm{mg} /$ day), where after initial control of inflammation with steroids, $42 / 54$ affected eyes improved or maintained Snellen visual acuity with CsA. In this group $31 / 54$ affected eyes presented with $6 / 12$ or worse vision, reflecting the severity and 'sight losing' nature of the inflammation which necessitated prolonged therapy for which long term steroid use alone would have required doses well within the toxic range.

ANTIMETABOLITES AND CYTOTOXIC AGENTS

Several different cytotoxic immunosuppressive agents have been used to treat EPU. These agents include azathioprine $^{334244}$ a purine antagonist, antimetabolite, which may be used either as an alternative to CsA in combination with steroids or in addition to CsA and steroid therapy. Methotrexate, which is a folic acid antagonist

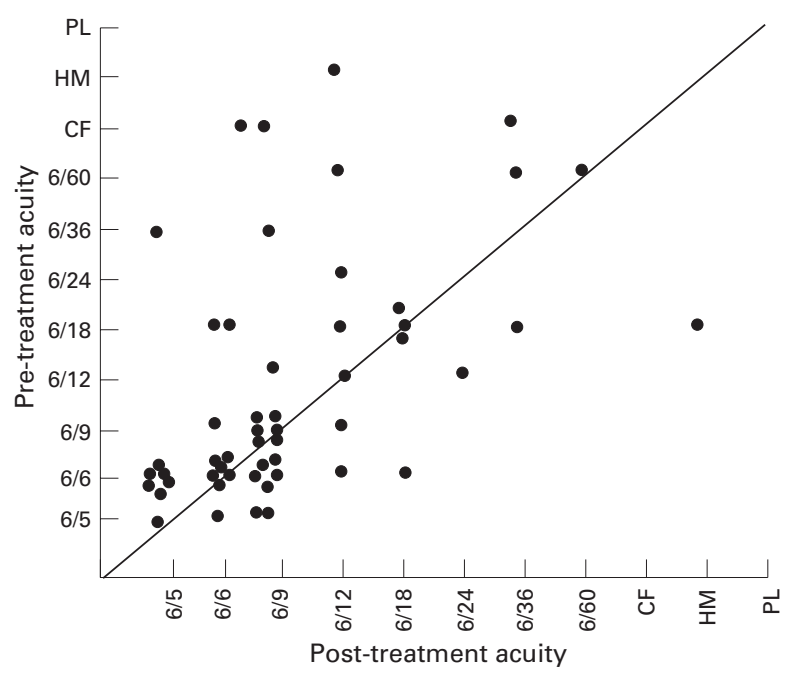

Figure 1 Recovery of visual acuity in a cohort of patients with EPU treated with a reducing course of prednisolone and maintenance of less than $10 \mathrm{mg} /$ day of prednisolone. All affected eyes are included in plot. In general there is visual recovery or vision is protected. $36 / 53$ eyes showed improvement in vision or maintained visual acuity (20 eyes and 16 eyes respectively) and only 6/53 eyes suffered a visual loss of greater than 2 lines of Snellen vision. 


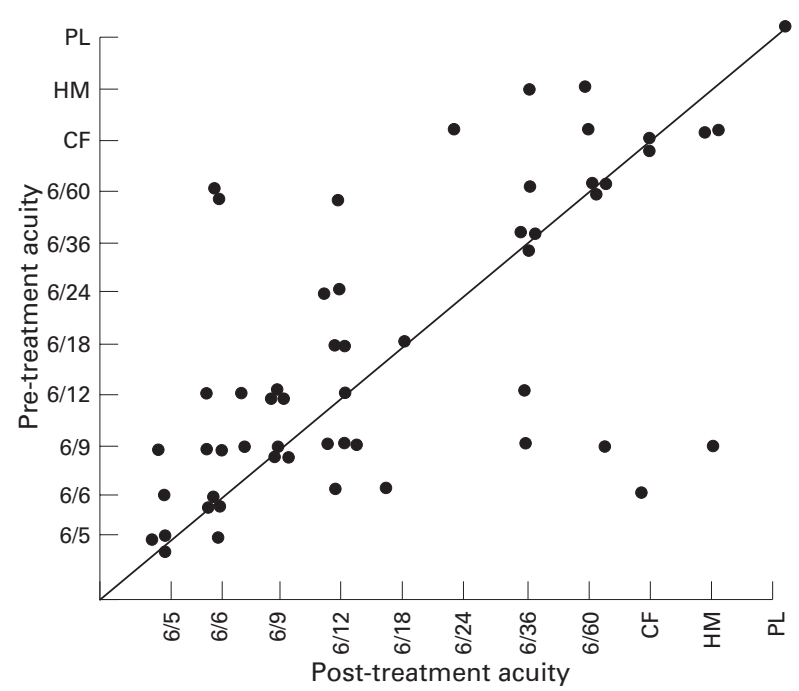

Figure 2 Recovery of visual acuity in a cohort of patients with EPU treated with combined low dose Cs $A$ ( $<5 \mathrm{mg} / \mathrm{kg} /$ day) and prednisolone $(<10 \mathrm{mg} /$ day). All affected eyes are included in plot. The vision of 21/54 eyes improved with this therapy, and a further $20 / 54$ eyes maintained their presenting vision. 10/54 eyes still had a visual acuity loss of greater than 2 lines of Snellen vision.

antimetabolite cytotoxic agent, has been used with some success in the treatment of EPU as weekly doses ${ }^{43}{ }^{45}$ either in combination with prednisolone or as part of triple therapy with CsA and prednisolone. Other more powerful cytotoxic immunosuppressants include the alkylating agents - for example, cyclophosphamide and chlorambucil. Cyclophosphamide is generally restricted to cases of severe refractory uveitis or cases where there are signs of ANCA positive systemic vasculitis with deteriorating organ function. ${ }^{46}$ Chlorambucil is rarely used in the UK for the treatment of EPU, and its effectiveness has been questioned. ${ }^{47}$ Newer agents which have been used in the treatment of EPU, although not universally, include bromocriptine. ${ }^{48}$ Bromocriptine augments the effect of CsA therapy, their synergistic immunosuppressive action being mediated by blockade of prolactin receptors on $\mathrm{T}$ cells and thereby inhibiting proliferation. ${ }^{49}$

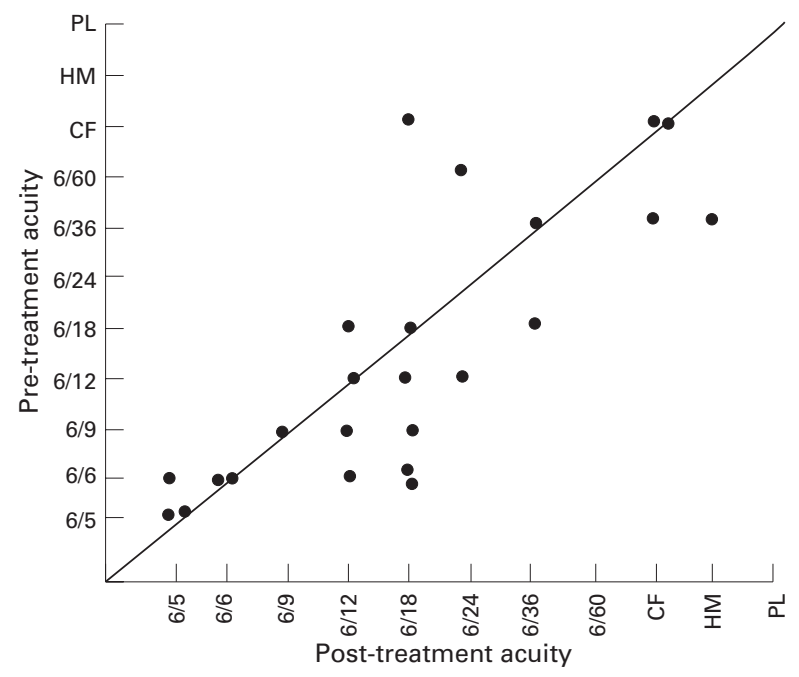

Figure 3 Recovery of visual acuity in a cohort of patients with EPU treated with combined low dose CsA ( $<5 \mathrm{mg} / \mathrm{kg} /$ day) and azathioprine (1-3 mg/kg/day) and variable maintenance doses of oral prednisolone. All affected eyes are included in plot. The inflammation in 14/24 eyes was able to be controlled so that the vision either improved (4/24) or was maintained (10/24). However, the vision in 10/24 eyes did deteriorate despite triple therapy.
Table 2 Baseline investigations for systemic immunosuppression in the treatment of endogenous posterior uveitis

Haematological Full blood count and differential

Biochemical Creatinine and urea, random glucose, creatinine clearance, and $/{ }^{51} \mathrm{Cr}$ glomerular filtration rate assessment

Liver function tests

Cardiopulmonary Chest $x$ ray, blood pressure assessment, ECG

Genitourinary Midstream urine/urinalysis and microscopy

Figure 3 demonstrates the response to treatment vis a vis visual acuity, in patients with severe EPU before and after triple therapy with azathioprine $(1-3 \mathrm{mg} / \mathrm{kg} /$ day $), \mathrm{CsA}(<5$ $\mathrm{mg} / \mathrm{kg} / \mathrm{day})$, and prednisolone. These patients represent those who 'failed' therapy with prednisolone alone or with prednisolone and CsA - that is, those below the line in Figures 1 and 2 . Pretreatment visual acuity was generally poor in these patients with $18 / 24$ eyes having $6 / 12$ or worse vision. Triple therapy controlled the inflammation in $14 / 24$ affected eyes, and maintained vision in $10 / 24$ with improvement in vision in $4 / 24$ eyes. It can be seen, therefore, that over $50 \%$ of such patients can be rescued from inexorable visual loss by using triple therapy. There still remains a small group who failed with this therapy.

\section{Treatment guidelines}

It will be clear from the above that a simple general guideline or protocol for the treatment of sight threatening EPU is possible, given our understanding of the mechanism of the disease and how the drugs act. Such a protocol is detailed below and has evolved from our experience in the treatment of over 500 patients with EPU. Before embarking on the protocol, however, a number of investigations are necessary. Investigations in patients with EPU are performed for two reasons: (i) to determine the nature, cause, and severity of the disease, and (ii) to ensure that the patient is generally well enough to tolerate the immunosuppressive drug regimens. A guide to baseline investigations is detailed in Table 2 .

If there are no clinical or laboratory based contraindications or relative contraindications to immunosuppression (for example, uncontrolled diabetes for steroid use and poor renal function for CsA therapy), and immunosuppressive therapy is indicated on clinical grounds the following protocol for the induction of immunosuppression has been found to be effective.

(1) The acutelactive stage of disease is controlled with systemic steroids as primary therapy.

The first aim is to treat 'sight threatening' disease as soon as possible. This is best achieved using systemic corticosteroids at a dose of $0.5-1.0 \mathrm{mg} / \mathrm{kg} / \mathrm{day}$. The dose depends on severity and occasionally intravenous pulsed methylprednisolone ( $1 \mathrm{~g} /$ day for 3 days) may be required in order to gain control.

(2) Long term control is achieved with low dose Cs A therapy as primary therapy, with or without combined low dose steroid therapy.

The second aim of therapy is to maintain control using additional immunosuppressants. Effective control can be achieved with CsA at a dose of $5 \mathrm{mg} / \mathrm{kg} /$ day. However, at this dose maximal effect is not achieved for 7-14 days and it is important therefore to continue an adequate steroid dose during this initial phase. Patients with mild disease (for definition see above) may be controlled on steroids alone if the maintenance dose is less than $10 \mathrm{mg} / \mathrm{day}$. However, monotherapy with low dose CsA can also adequately control mild disease.

Once effective control has been achieved (usually between 3-6 weeks after commencing therapy) the aim is to control inflammation on a minimally effective dose. Normally, this is achieved by tapering corticosteroids to a 
low or even zero dose (should not be greater than $10 \mathrm{mg}$ prednisolone) while gradually reducing CsA to $2-3 \mathrm{mg} / \mathrm{kg} /$ day over several weeks. This therapy can be maintained for several months to years if necessary (see below, monitoring drug therapy).

(3) Additional immunosuppressants are required when inflammation is present despite low dose therapy with Cs $A$ and steroids.

Certain patients who are inadequately controlled on the above therapy (for example, those requiring a maintenance dose of greater than $15 \mathrm{mg}$ /day of prednisolone), should be considered for additional immunosuppression. A commonly used third line drug, azathioprine (at $1-3 \mathrm{mg} / \mathrm{kg} /$ day), should be instituted. Other agents which can be considered include methotrexate $(1 \mathrm{mg} / \mathrm{kg} /$ week) and oral cyclophosphamide. These additional agents may be combined not only with prednisolone and CsA but also used separately with prednisolone only if there are contraindications to CsA therapy.

\section{Monitoring therapy: endeavouring to secure a successful outcome}

During the induction and maintenance periods of immunosuppressive therapy, close attention to 'outcomes' will ensure that control is not lost while attempting to achieve the lowest effective dose.

MONITORING THE THERAPEUTIC RESPONSE

This is achieved by:

- subjective and objective assessment of visual acuity and/or visual field

- objective assessment of the level of inflammation using a standardised scoring system. ${ }^{22}$ This comprises (a) an evaluation of the degree of vitreal haze with the binocular indirect ophthalmoscope (BIO) as a BIO score (0-5) (see BenEzra et $\mathrm{al}^{22}$; (b) an assessment of the area of retinochoroidal involvement with active choroiditis and vasculitis lesions; and (c) an evaluation of the severity of macular and optic disc oedema.

Patients should be first reassessed 2 weeks after induction of immunosuppression and thereafter at 4 week intervals until stability is achieved. After a period of at least 3 recurrence free months have passed, immunosuppressive therapy may be tapered further, to the point of treatment cessation if no relapses occur. It is important to emphasise, however, that each patient must have a customised approach depending on their response to treatment.

\section{MINIMISING SIDE EFFECTS \\ Corticosteroids}

As Figure 1 shows we and others ${ }^{21}{ }^{37}$ have had success with steroid therapy alone, where after an initial high dose regimen of prednisolone $(1 \mathrm{mg} / \mathrm{kg} /$ day $)$ the steroid dose is reduced to a maintenance level of $<10 \mathrm{mg} /$ day over 3 months. Steroid induced side effects are, however, common if not the rule, and thus are not recommended for long term use at doses over 5-10 mg daily.

Side effects include a subclinical hypothalamicpituitary-adrenal axis dysregulation, weight gain, myopathy, abnormal glucose tolerance or diabetes, hypertension, accelerated arteriosclerosis, and atheroma. Weight gain, change in facial appearance, and acne are particularly disturbing to young people. Steroid induced osteoporosis leads to vertebral collapse and an increased incidence of fractures and avascular necrosis. ${ }^{50}$ These side effects are cumulative with prolonged therapy. The American Council of Rheumatologists (ACR) reports that long term use of prednisolone at doses greater than $7.5 \mathrm{mg} /$ day can cause
Table 3 Monitoring of immunosuppressant therapy in endogenous posterior uveitis

\begin{tabular}{ll}
\hline Corticosteroids & - Weight, general habitus including skin changes \\
& - Urinalysis and random blood glucose (fasting blood \\
glucose and/or glucose tolerance test if indicated) & - Blood pressure \\
& - Electrolytes (hypokalaemia) \\
& - (Bone densitometry) \\
Cyclosporin A & Blood pressure \\
& - Creatinine, blood urea nitrogen, and regular glomerular \\
& - Liltration rate assessment for renal toxicity \\
& - Electrolytes, including magnesium \\
& - Full blood count (particularly lymphopenia) \\
& - Full blood count and differential for leucopenia (in \\
Azathioprine & particular neutropenia). Anaemia, thrombocytopenia, \\
& and leucoerythroblastic anaemia occur more rarely
\end{tabular}

significant bone loss and that most patients have low bone mineral density accounting for $25 \%$ of fractures in groups of patients. ${ }^{51}$ The ACR recommends that those patients who are at high risk should be counselled and appropriate prophylaxis considered and instituted. Mild to severe psychoses are also very common and are significant reasons for reluctance in restarting steroids when relapses occur. Patients who have stopped steroid therapy frequently announce their relief at their freedom from symptoms.

\section{Cyclosporin A}

There is also a concern in ophthalmic practice about the side effects of CsA therapy which has restricted its use, despite its well documented clinical benefit and efficacy in the treatment of EPU. ${ }^{30-33} \mathrm{CsA}$ induced nephrotoxicity is dose dependent and may occur even at low doses. Nearly all the patients who commence CsA therapy show biochemical evidence of a proximal tubulopathy which is reversible on dose reduction..$^{53}$ The glomerular filtration rate may also be reduced with long term therapy, and not always associated with a rise in serum creatinine. ${ }^{53}$ The risk of nephrotoxicity is increased with pre-existing hypertension and with the use of certain drugs such as non-steroidal anti-inflammatory drugs (NSAIDS). Importantly, in one study no significant nephrotoxicity or hypertension was found in young patients (under 30 years) who had systemic associated disease. ${ }^{31}$ Hepatotoxicity is an unusual complication in Europe and in our experience rarely raises liver enzymes to necessitate cessation of CsA.

Lifetime treatment of transplant patients has shown that CsA therapy is associated with a risk of malignancy, particularly lymphomas. However, the risk in using low dose CsA in uveitis is likely to be low, in part because treatment of EPU with CsA is often a short to mid term therapy ( $<2$ years) and partly because of the lower doses used.

Control of hypertension in steroid-CsA treated EPU patients is best achieved both by conservative measures including weight loss and reduction in alcohol intake, and by reducing the dose of immunosuppressive agents, in particular high dose corticosteroids in the first instance. CsA therapy can still be given safely to controlled hypertensives, provided renal function is regularly monitored. Blood pressure control is best achieved with calcium antagonists because of its renal protective effect when patients are receiving $\mathrm{CsA}^{54}$ allowing maintenance of renal function without modulating CsA dose.

It is important to inquire at each visit to the clinic using a simple check list whether the patient has any specific symptoms and signs which might indicate side effects. This should be combined with regular laboratory tests specific for the individual drugs. The common features which require attention are detailed in Table 3. Lastly, it is important to recognise when treating these patients that there are many drug interactions which can increase the toxicity of 
the immunosuppressant. In addition, one must avoid combining cytotoxic agents which are myelosuppressive.

\section{The next generation of immunosuppressants}

While many cases of EPU are adequately controlled on the above regimen, particularly if carefully monitored to achieve the lowest effective dose of drugs in combination, some patients will escape control. Such patients are either refractory to therapy or more commonly develop intolerable side effects. Newer immunotherapeutic approaches are available and are currently under evaluation and should be considered. These include FK $506,{ }^{55}$ mycophenolate mofetil, ${ }^{56}$ and inhibition of the effector immune response with monoclonal antibodies against specific lymphocytes and proinflammatory cytokines. ${ }^{22}{ }^{25}$ Hopefully, these will allow safer and more precise control of inflammation in EPU and thus reduce the morbidity for this disease. ${ }^{27}$

ANDREW D DICK MOHAMMED AZIM JOHN V FORRESTER

Department of Ophthalmology, Medical School, University of Aberdeen, Foresterhill, Aberdeen AB9 2ZD

Correspondence to: Dr Andrew D Dick.

1 Forrester JV. Uveitis: pathogenesis. Lancet 1992;388:1498-501.

2 Rothova A, Suttorp-Schulten MSA, Treffers WF, Kijlstra A. Causes and frequency of blindness in patients with intraocular inflammatory disease. $\mathrm{Br}$ 7 Ophthalmol 1996:80:332-6.

3 Couto C, Merlo JL. Epidemiological study of patients with uveitis in Buenos Aires, Argentina. In: Dernochamps JP, Verougstraete C, Caspers-Velu 1, Tassignon MJ, eds. Recent advaces in uveitis. Amsterdam: Kugler, 1993:171-4.

4 Whitcup SM, Salvo EM, Nussenblatt RB. Combined cyclosporine and corticosteroid therapy for sight threatening uveitis in Behçet's disease. $A m \mathcal{F}$ Ophthalmol 1994;118:39-45.

5 Towler HMA, Lightman S. Visual prognosis in Behçet's disease. Ocul Immunol Inflamm 1993;1:249-54.

6 Chisolm IH, Gass JDM, Muuton WL. The late stage of serpiginous (geographic) choroiditis. Am F Ophthalmol 1976;82:343-51

7 Suttorp-Schultern, Rothova A. The possible impact of uveitis in blindness: a literature survey. Br f Ophthalmol 1996;80:844-9.

8 Rothova A. Ocular involvement in toxoplasmosis. Br f Ophthalmol 1993;77: 371-7.

9 Forrester JV. Endogenous posterior uveitis. Br f Ophthalmol 1990;74:620-3.

10 Forrester JV, Lumsden L, Liversidge J, Kuppner M, Mesri M. Immunoregulation of uveoretinal inflammation. Prog Ret Res 1995;14:393-411.

11 Forrester JV. Duke Elder Lecture: new concepts on the role of autoimmunity in the pathogenesis of uveitis. Eye 1992;5:433-47.

12 Forrester JV, Liversidge J, Dua HS, Towler H, McMenamin PG. Comparison of clinical and experimental uveitis. Curr Eye Res 1990;9:75-84.

13 Caspi RR, Roberge FG, McAllister CG, El-Saied M, Kuwabara T, Gery I, et al. T cell lines mediating experimental autoimmune uveoretinitis (EAU) in al. $T$ cell lines mediating experimenta
the rat. F Immunol $1986 ; 136: 928-33$.

14 Dick AD. Retinal antigen-specific T cells mediate experimental autoimmune uveoretinitis (EAU) in PVG rat. Ocul Immunol Inflamm 1995;3:26170 .

15 Nussenblatt RB, Rodrigues MM, Walker WB, Cevario SJ, Salinas-Carmara MC, Gery I. Cyclosporin A: inhibition of experimental autoimmune uveitis in Lewis rat. $\mathcal{F}$ Clin Invest 1981;67:1228-332.

16 Dick AD, Cheng YF, Purdie AT, Liversidge J, Forrester JV. Immunocytochemical analysis of blood lymphocytes in uveitis. Eye 1992;6:643-7.

17 Arocker-Mettinger E, Steurer-Georgiew L, Steure M, Huber-Spitsy V, Hoetzl E, Grabner G, et al. Circulating ICAM-1 levels in serum of uveitis patients. Curr Eye Res 1992;11:161-6.

18 Nussenblatt RB, Gery I, Weiner, H, Ferris FL, Shiloach J, Remaley V. Treatment of uveitis by oral administration of retinal antigens:results of a phase I/II randomised masked trial. Am f Ophthalmol 1997;123.583-92.

19 Nussenblatt RB, Palestine AG, Chan CC. Cyclosporin A therapy in the treatment of intraocular inflammatory disease resistant to systemic corticosteroids and cytotoxic agents. Am f Ophthalmol 1983;96:275-82.

Lightman S. Uveitis: management. Lancet 1991;338:1501-4.

20 Lightman S. Uveitis: management. Lancet 1991;338:1501-4.
21 Lightman S. Use of steroids and immunosuppressive drugs in the management of posterior uveitis. Eye 1991;5:294-9.

ment of posterior uveitis. Eye 1991;5:294-9.
22 BenEzra D, Forrester JV, Nussenblatt RB, Tabbara K, Timonen P. Uveitis scoring system. Berlin: Springer-Verlag, 1992:1-9.

23 Dick AD. Experimental approaches to specific immunotherapies in autoimmune disease: future treatment of endogenous posterior uveitis. $\mathrm{Br} \mathcal{F} \mathrm{Oph}$ thalmol 1995;79:81-8.
24 Rizzo LV, Caspi RR. Immunotolerance and prevention of ocular autoimmune disease. Curr Eye Res 1995;14:854-64.

25 Isaacs J, Dick AD. Short term immunosuppressive therapy and long term immunoregulation:promises and problems. $\mathrm{Br} f$ Ophthalmol 1996;80: $1035-7$

26 Waldmann $\mathrm{H}$, Cobbold S. The use of monoclonal antibodies to achieve immunological tolerance. Immunol Today 1993;14:247-51

27 Isaacs JD, Watts RA, Hazleman BL, Hale G, Keogan MT, Cobbold SP, et al. Humanised monoclonal antibody therapy for rheumatoid arthritis. Lancet 1992;340:748-52.

28 Elliott MJ, Maini RN, Feldman M, Long-Fox A, Charles P, Katsikis P, et al. Treatment of rheumatoid arthritis with chimeric monoclonal antibodies to tumour necrosis factor alpha. Arthritis Rheum 1993;12:1681-90.

29 Gordon DM. Prednisone and prednisolone in ocular disease. Am f Ophthalmol 1956;41:593-600.

30 Towler H, Cliffe AM, Whiting P, Forrester JV. Low dose cyclosporin A and steroid therapy. Eye 1989;3:282-7.

31 Towler HM, Whiting PH, Forrester JV. Combination of low dose cyclosporin $\mathrm{A}$ and steroid therapy for chronic intraocular inflammation. Eye $1990 ; 4: 514-20$.

32 Vitale AT, Rodriguez A, Foster CS. Low dose CsA therapy in treating chronic intraocular non-infectious uveitis. Ophthalmology 1996;163:635-

33 Towler HM, Forrester JV, Lightman S. Low dose CsA therapy of ocular inflammation: preliminary report of a long term follow up study. Autoimmunity 1992;5:259-64.

34 Helm CJ, Holland GN. The effects of posterior subtenon injection of triamcinolone acetonide in patients with intermediate uveitis. Am f Ophthalmol 1995;120:55-65.

35 Russin MM, Tessher HH, Cunha-Vas JG. Posterior subtenon injection of corticosteroids in uveitis patients with cystoid macular oedema. $f_{p n} \mathcal{F} O p h-$ thalmol 1988;34:385-91.

36 Weijtens O, van der Sluijs FA, Schoemaker RC, Lentjes EG, Cohen AF, Romijn FP, et al. Peribulbar corticosteroid injection: vitreal and serum concentrations after dexamethasone disodium phosphate injection. Am $7 \mathrm{Oph}$ centrations after dexameth $1997 ; 123: 358-63$.

37 Howe LJ, Stanford MR, Edelsten C, Graham EM. The efficacy of systemic corticosteroids in sight-threatening retinal vasculitis. Eye 1994;8:443-8.

38 Nussenblatt RB, Palestine AG, Chan CC, Stevens G, Mellow SD, Green SB. Randomised double masked study of cyclosporine compared to prednisolone in the treatment of endogenous uveitis. Am $\mathcal{f}$ Ophthalmol 1990;112:138-46.

39 Calne RY. Immunosuppression for organ-grafting: observations on cyclosporin A. Immunol Rev 1979;46:113-28.

40 Liu J. FK 506 and cyclosporin, molecular probes for studying intracellular signal transduction. Immunol Today 1993;14:290-6.

41 Granelli-Piperno A. In situ hybridisation for IL-2 and IL-2R mRNA in T cells activated in the presence of CsA. F Exp Med 1988;5:1649-58.

42 Hooper PL, Kaplan HJ. Triple agent immunosuppresion in serpiginous choroiditis. Ophthalmology 1991;98:944-50.

43 Pascalis L, Pia G, Aresu G. Combined cyclosporin-steroid-methotrexate treatment in endogenous non-infectious uveitis. F Autoimmun 1993;6:46780

44 Yazici H, Pazarli H, Barnes CG. A controlled trial of azathioprine in Behçet's syndrome. N Engl f Med 1990;322:281-5.

45 Shah SS, Lowder CY, Schmitt MA. Low dose methotrexate therapy for ocular inflammatory disease. Ophthalmology 1992;99:1419-32.

46 Rosenbaum JT. Treatment of severe refractory uveitis with intravenous cyclophosphamide. F Rheumatol 1994;21:123-5.

47 Tabbara KF. Chlorambucil in Behçet's disease. Ophthalmology 1983;90: 906-8.

48 Palestine AG, Nussenblatt RB, Gelato $M$. Therapy for autoimmune uveitis with low-dose cyclosporine plus bromocriptine. Transplant Proc 1988;20: 131-6.

49 Palestine AG, Muellenberg-Coulombre CG, Klim MK, Gelato MC, Nussenblatt RB. Bromocriptine and low dose cyclosporine in the treatment of experimental autoimmune uveitis in the rat. $\mathcal{F}$ Clin Invest 1987;79:107881 .

50 Walsh LJ, Wang CA, Pringle M, Tattersfield AE. Use of corticosteroids in community and prevention of secondary osteoporosis: a cross sectional community and prevention
study. $B M \mathcal{F} 1996 ; 313: 344-6$.

51 Holchberg MC, Prashker MJ, Greenwald M, Hannan MT, Lane NE, Lindsey SM, et al. Recommendations for the prevention and treatment of glucocorticoid-induced osteoporosis. Arthritis Rheum 1996;39:1791-801.

52 Whiting PH, Towler HMA, Cliffe AM, Forrester JV. The use of lithium clearance measurements to assess renal tubular function in cyclosporin treated uveitis patients. Lithium 1990;1:245-50.

53 Dick AD, Towler HMA, Whiting P, Forrester JV. The use of lithium clearance studies in the early detection of cyclosporin A (CsA) nephrotoxicity: a protocol of renal function assessment with CsA therapy. Curr Eye Res 1992. 11 (Suppl):215-8.

54 McNally PG, Walls J, Feehally J. The effect of nifedipine on renal function in normotensive cyclosporin A treated renal allograft patients. Nephrol Dial Transplant 1990;5:926-8.

55 Ishioka M, Ohno S, Nakamura S, Isobe $\mathrm{K}$, Watanabe N, Ishigatsubo Y, et al. FK 506 treatment of non-infectious uveitis. Am $\mathcal{F}$ Ophthalmol 1995;118: $723-30$

56 Chanaud NP, Vistica BP, Eugui E, Nussenblatt RB, Allison AC, Gery I. Inhibition of experimental autoimmune uveoretinitis by mycophenolate mofetil, an inhibitor of purine metabolism. Exp Eye Res 1995;61:429-34. 